\title{
Correction to: Fractured Reservoirs Modeling by Embedded Fracture Continuum Approach: Field-Scale Applications
}

Hong-Lam Dang, Duc-Phi Do, and Dashnor Hoxha

\begin{abstract}
Correction to:
Chapter "Fractured Reservoirs Modeling by Embedded Fracture Continuum Approach: Field-Scale Applications" in: M. F. Randolph et al. (Eds.): Proceedings of the 1st Vietnam Symposium on Advances in Offshore Engineering, LNCE 18, https://doi.org/10.1007/978-981-13-2306-5_15
\end{abstract}

The affiliation "Civil Engineering Faculty, University of Transport and Communication, Hanoi, Vietnam" of author "Hong-Lam Dang" in the original version of the book has been changed to "University of Transport and Communications, No.3 Cau Giay Street, Hanoi, Vietnam" in the chapter "Fractured Reservoirs Modeling by Embedded Fracture Continuum Approach: Field-Scale Applications". This has been corrected in the updated version. 